\title{
Driving Faster Than a Human Player
}

\author{
Jan Quadflieg, Mike Preuss, and Günter Rudolph \\ Chair of Algorithm Engineering, Computational Intelligence Group, \\ Dept. of Computer Science, Technische Universität Dortmund, Germany \\ firstname. lastname@tu-dortmund. de
}

\begin{abstract}
TORCS car racing bots have improved significantly in the last years. We show that accurate curvature information for the upcoming corners enables offline learning a near-optimal driving style that consistently beats an expert human player (and the fastest currently known bots). Generalization to other tracks often, but not always succeeds, so that the method is extended by an online error correction mechanism well suited to the warmup phase of the Simulated Car Racing Championships.
\end{abstract}

\section{Introduction}

Is it possible to establish a TORCS bot that consistently drives faster than an expert human player? The last years of bot development for The Open Racing Car Simulator have seen considerable improvement, namely the Cobostar [2] bot, Onieva and Pelta's bot [7], Jorge Munoz' bot [6] and Autopia, for an overview also see [5]. While opponent handling, especially overtaking, still remains a largely unresolved issue, progress has been made in single car driving, gradually shifting from ad-hoc actions in response to incoming sensor values to a more global view that enables planning racing lines, as e.g. demonstrated by [3] and [8]. The latter work also contained an overview of round times attained by a moderately trained human which in many cases exhibited a considerable potential of improvement as the existing bots in most cases did not manage to come close to the human achievement.

However, there is no reason why a computer controlled bot shall not be able to close this gap. We argue that two factors are needed to do so: a) accurate processing of sensory information in order to correctly predict the curvature of the upcoming corner, and b) knowledge of the whole track in terms of a track model. Both is possible in principle with the methods provided in [8]. This holds true only for the noiseless case, with sensor value noise, these methods unfortunately break down. We therefore only consider noiseless sensors in this work and show how the available information can be used to implement a driving style that goes to the limit-and sometimes beyond. Our basic idea is to use only curvature estimation of a corner for setting a target speed, and to use two modifiers that reduce the effect of acceleration and braking in dependence of the current steering angle (braking or accelerating fully may not be wise while holding the steering wheel at a $45^{\circ}$ position). For each of these three functional relationships, we may make the general assumption of monotony which enables us to use an evolutionary algorithm (EA) for fitting simple models during offline learning (section 2). The effect is tremendous. However, the learned behavior does not always generalize so that we

C. Di Chio et al. (Eds.): EvoApplications 2011, Part I, LNCS 6624, pp. 143-152, 2011.

(c) Springer-Verlag Berlin Heidelberg 2011 
add some online learning which adapts the driving style to the topology of a given track (section 3). Finally, we compare the resulting bot to the current best controllers and an expert human driver (section 4) and give conclusions and outlook.

Since TORCS has been used during the last three years in the context of the Simulated Car Racing Championship, we assume that most readers are familiar with the provided interface. If this should not be the case, we refer to the official manual [4] which contains an in depth description of the sensors and actuators which are available to a TORCS bot.

\section{Learning Target Speeds}

In [8], Quadflieg et al. presented a measure for the curvature of a race track, calculated from the 19 track sensors. Instead of using only the longest sensor value for detecting curvature as many other approaches do, this measure takes in much more data, helping to make it somewhat less dependent of the car direction and lateral track position. Quadflieg et al. map the measure to five discrete types of segments, from which a model of the race track is built during the first lap while driving on a previously unknown track. Target speeds for the four possible corner types and some other parameters were learned with an evolutionary algorithm.

However, this approach exhibited some problems due to the chosen discretization into different track segment types. As the corners within one type still possess some variance, the rough categorization led to a rather defensive driving behavior. On the level of a planning module, few categories thus make sense, but target speeds for one specific corner need to be dealt with more fine-grained to achieve competitiveness to the current best controllers and also human drivers.

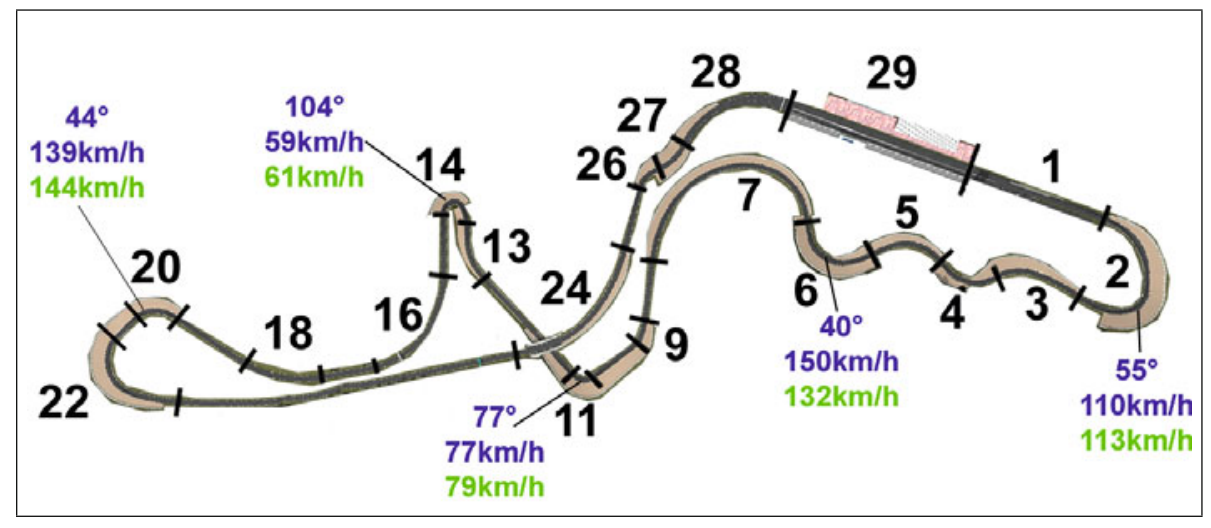

Fig. 1. Birds eye view of the track Wheel 2 (Suzuka). The numbers indicate the different segments of the track model as corresponding to the track definition (numbers of some straight segments have been omitted for the sake of clarity). For some selected corners we show the recorded maximum absolute value of the curvature measure (blue), the target speed, calculated with the learned logistic function (blue) and the target speeds further adapted with the online learning process (green, see section 3). 


\subsection{The Possible Speed is a Function of Curvature}

In opposite to the approach of [8], we here model the target speed directly as a function over the curvature measure. For every part of the track, we save the maximum absolute value of the measure encountered while building the track model. Figure 1 shows the track Wheel2, the segments of the learned track model and the maximum value of the curvature measure (coloured blue) for some selected corners. The measure is roughly zero on straight parts of the track, $40^{\circ}$ in medium speed corners like the Esses and $104^{\circ}$ at the hairpin. Under the curvature, target speeds (blue) are depicted as calculated by the adapted function that is described in the following.

As function template we choose a generalized logistic function, also named Richards curve [9], as it allows high flexibility, even asymmetry, while keeping monotonicity:

$$
\nu_{p}(\rho)=K-\frac{K-A}{\left(1+Q * e^{-B *(\rho-M)}\right)^{\frac{1}{v}}}
$$

where $\rho$ is the measure for curvature and $\nu_{p}$ the resulting target speed. The lower asymptote $A$ is set to $50 \mathrm{~km} / \mathrm{h}$, the upper asymptote $K$ to $330 \mathrm{~km} / \mathrm{h}$. This leaves us with four parameters to adapt: $B, Q, v$ and $M$.

While driving, the actual output of the controller for acceleration $a$ and brake $b$ is simply:

$$
a= \begin{cases}1 & \nu_{t} \leq \nu_{p} \\ 0.2 & \nu_{t}>\nu_{p} \wedge \nu_{t} \leq \nu_{p}+20 \mathrm{~km} / \mathrm{h} \\ 0 & \nu_{t}>\nu_{p}+20 \mathrm{~km} / \mathrm{h}\end{cases}
$$

and

$$
b= \begin{cases}0 & \nu_{t} \leq \nu_{p}+20 \mathrm{~km} / \mathrm{h} \\ 1 & \nu_{t}>\nu_{p}+20 \mathrm{~km} / \mathrm{h}\end{cases}
$$

where $\nu_{t}$ is the current speed of the car. This simple "binary" reaction proved to be rather dangerous, as it often causes the car to spin out of control.

To avoid this effect, we use two functions to dampen the output depending on the steering value determined by the steering module. The new output is the old one, multiplied with the damping function. These damping functions are also modeled as generalized logistic functions with the upper asymptote $K$ set to 1 and the lower asymptote $A$ set to 0 . The other parameters $(B, Q, v$ and $M)$ are included into the learning process, too, which leads to an amount of twelve real-valued variables we want to adjust.

Learning took place on the track Wheel2, because it contains virtually every type of corner one can imagine, using the well known CMA-ES [1]. The fastest lap time served as the fitness function, which we want to minimize. For one fitness evaluation, each candidate solution drove two laps, which takes roughly four and a half minutes for mediocre solutions. The evaluation was aborted after six minutes to avoid being stuck with a bad solution for too long. In this case, the fitness value was either set to the time of the first lap, or to six minutes if the car did not even finish one lap. Driving two laps enables one lap with flying start in opposite to a standing start, so the fastest possible round time with a certain driving behavior can be achieved. The track model had been recorded in advance, and thus was already available to the controller during the fitness evaluations. 

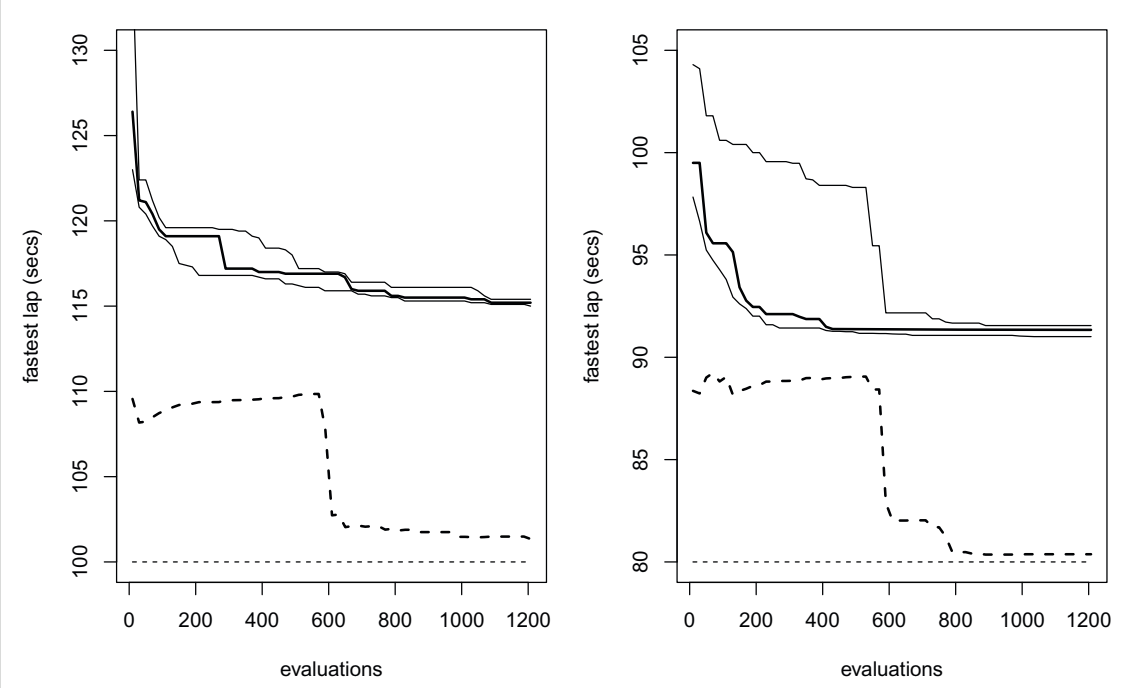

Fig. 2. Adaptation of our controller to the Wheel2 (left) and the Forza track (right), the upper curves show the median (thick) and upper and lower quartile lines (thin) of the achieved round times. The lower curves depict the standard deviations between the runs in the same scaling, the thin line standing for 0 .

\subsection{Experimental Results of the Offline Learning Process}

Next to presenting examples for the finally obtained solutions, we also need to tackle the research question if the chosen optimization method (CMA-ES) and the employed representation reliably lead to satisfying results.

Pre-experimental planning. Initial runs led to the insight that slightly increased population sizes (set to $(10,20)$ instead of the default values of $(5,10)$ ) may provide more reliability, and that initial stepsizes shall be chosen slightly smaller than usual ( 0.1 here). Run length is chosen to 1200 as longer runs result in little additional progress.

Task. A round time in the range of an excellent human driver $(\approx 115 \mathrm{~s}$ for Wheel 2 and $\approx 92 \mathrm{~s}$ for Forza) has to be achieved in all runs.

Setup. We run the CMA-ES with the specific parametrization given above 7 times on the Forza and Wheel 2 tracks, respectively (one run takes about a day on a modern 4-core machine, allowing up to 2 laps or 6 mins max. for each evaluation).

Result/Visualization. Figure 2 document the optimization progress on two tracks, Wheel 2 and Forza, figure 3 shows the best overall solution for the track Wheel2.

Observations. The overall progress of the optimization is similar in both cases, very remarkably is the breakdown of the standard deviation around evaluation 600 . For Wheel2, optimization still makes progress at the end, whether for Forza, stagnation seems to be reached. 


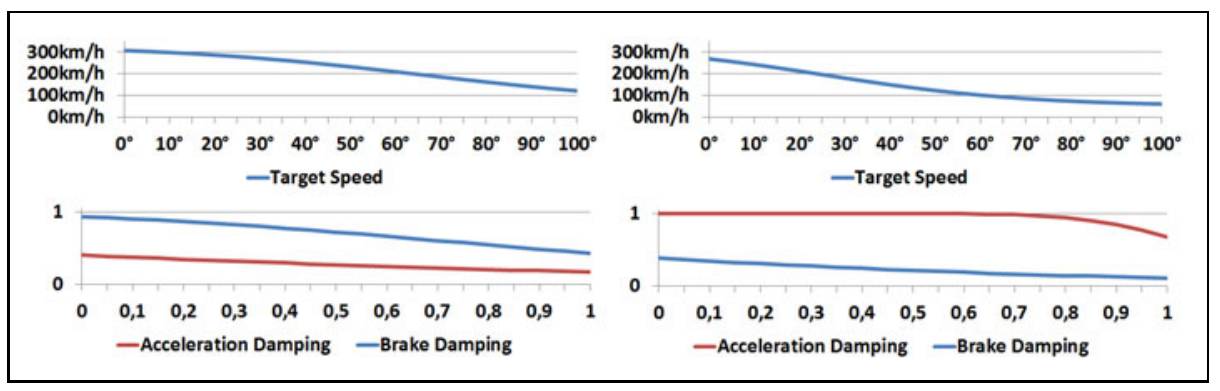

Fig. 3. Visualization of the obtained solutions. The left plots show the best solution from the start population of run six on wheel2, with has a fitness value of 127.8. The right plots shows the best individual from run six, which is also the overall best solution, with a fitness value of 114.8.

Discussion. The downturn of the standard deviation may stem from different run lengths until the optimization detects a good area. Note that if a restart was performed due to stagnation, it happened mostly around evaluation 600 on Wheel2, but less often and later for Forza. The high standard deviation on Forza hints to the fact that obtaining good starting individuals may be more difficult on this track. On the other hand, the track has fewer different features if compared to Wheel2, so that the optimization potential shall be smaller.

The overall aim of reliably reaching human-competitive round times is achieved, interestingly best solutions from the single runs can be quite different in structure while being very similar in quality. However, note that we do not state that the chosen optimization method cannot be improved. E.g., parameter tuning could be applied to make it a bit faster, or the representation could be changed slightly to get rid of redundancies.

\section{Online Adaption of the Learned Behaviour}

First tests on other tracks showed that the learned behaviour doesn't always generalize well. More concrete, the controller runs into difficulties when confronted with situations that deviate considerably from what he was trained on, e.g. if the friction of the track decreases (Brondehach and Monandgo), or a track has no driveable margins (Alpine-1). Luckily, the current format of the simulated car racing championship contains a warmup stage which can be used to fine tune the learned behaviour. To take advantage of this, our controller contains a simple online learning module, which consists of four phases:

Phase 1. In the first phase our controller drives one lap with a constanst speed of $50 \mathrm{~km} / \mathrm{h}$ to learn the track model. When the track model is complete, the target speeds for all the corners are initialized to default values calculated with the learned logistic function.

Phase 2. In the second phase, the controller tests the default target speeds. If it leaves the track or gets more than 50 points of damage, the target speeds of the corresponding track segment and the one before are lowered by $20 \%$. Besides that, the segments are marked as being "problematic". Lowering the target speeds by $20 \%$ is a rather defensive approach, but the goal of the second learning phase is to reach a 
save state as fast possible. Presumably, only around 15 rounds can be driven during the whole warmup phase, so that not much of this small budget shall be lost here. As soon as the controller is able to complete two laps without leaving the track, it switches to the third phase.

Phase 3. Knowing that $20 \%$ decreases are very large steps, the target speeds for problematic corners are increased again in the third phase. First by $10 \%$ and if that doesn't work for a specific corner by $5 \%$ or $2.5 \%$, respectively. As soon as the controller can drive two consecutive laps without leaving the track or taking damage, it switches to the final phase.

Phase 4. The objective of this phase is to attempt minimal tuning also for the yet untuned cornders. The target speeds for all corners not marked as being problematic are increased by $1 \%$. If the car is able to drive two laps without leaving the track, this is done again. If the car leaves the track, the target speed of the corresponding segment is reset to the old value and the corner is excluded from the optimization. This is done until the controllers runs out of time.

Note that we do not take the actual lap times into account during the online optimization. Due to the small time budget, we have to act on all pieces of information that can be obtained within a round. We only lower speeds when we get in trouble and increase the speeds later on in the hope that this makes us faster, which is not necessarily the case (when driving too fast, we leave the track again and get slower). This first online learning approach can surely be improved but works well for achieving controllers that stay on the track and drive very fast.

As it was claimed that our controller can drive faster than existing controllers and even than an experienced human driver, we need a comparison. As benchmarks we use the best controllers of the 2009 and 2010 championship: Cobostar (2nd place 2009), Autopia (winner 2010) and the contribution of Jorge Muñoz (2nd place 2010). All evaluations were done without noise, which should not be a disadvantage for the other controllers. Comparisons during the 2010 championship disclosed that all controllers are still faster without noise.

In [8] it was shown that on trivial tracks, like oval shaped speedways which can be driven flat out, the gap between the best controllers and a human player is close to non existing. We therefore compare on more demanding tracks, of which most are replicas of race tracks which exist in real live: Alpine-1, Forza and Wheel2, which are all included in the standard Torcs distribution [10]. These were supplemented with other tracks available online: Curburgring, Goldstone, Monandgo, Brondehach and Ardennen-Spa 1 .

The evaluation followed the format of the 2010 championship: Each controller was allowed to drive 100,000 gameticks (roughly 36 minutes) alone, to adapt to the given track. After that, each controller drove for five consecutive laps. Since Cobostar is a 2009 controller which cannot take advantage of the warmup, Cobostar just drove for five laps on each track.

\footnotetext{
${ }^{1}$ Curburgring, Goldstone and Monandgo are available at

http: //apr-free.info/joomla/index.php/descargas/category/

2-circuitos-for1-torcs, Brondehach can be found at

http://sites.google.com/site/torcscreations/tracks and Spa at

http: / / t-xchange.co.cc/forum/viewtopic.php? f=9\&t=24
} 


\section{Experimental Comparison}

Figure 4 depicts the time allocated for the different phases of our online learning algorithm on the different tracks. Since the car drives with a constant speed during the first phase, the time needed to record the track model depends entirely on the length of the race track. The only exception to this was the Curburgring where our controller went off the track and needed two laps to record the track model. The track model for Wheel 2 used for the offline learning described in section 2 was deleted and had to be recorded again for the evaluations to match the situation on the other tracks.

On the tracks Curburgring, Monandgo, Brondehach and Spa, the controller was unable to reach a save state and was therefor stuck in the second phase: It continued to lower target speeds for certain corners until it finally ran out of time. While it is unclear why this happened on the Curburgring, the other three tracks revealed a false assumption in our naiv approach to adapting the behaviour: We only thought of losing control because the car drives too fast. But in these cases, the steering is a bit too aggressive and the car leaves the track on the inside of a corner. Lowering the target speed for this corner does not help, so that the controller is unable to adapt its behaviour in the right direction. On Goldstone, the learned behaviour works well and the second phase only lasts for two laps, which can be driven without problems. For that reason, the third phase could actually be skipped but since we did not take this possibility into account, the controller spends some time doing nothing until it reaches the final phase and slowly increments the target speeds of all corners until it runs out of time. In total, the target speeds could be incremented by $5 \%$ without any problems, so the performance might get even better with a longer warmup. The situation is nearly identical on the remaining tracks Wheel2, Forza and Alpine-1, although the latter proved to be difficult and the controller needed very much time to get to a save state.

Figure 5 shows the comparison with other controllers and a human player, who is an experienced player of car racing simulations. Nethertheless he was unable to drive on the track Monandgo, reporting that he "couldn't see anything but different shades of grey", which has to do with the lack of details in the currently available $3 \mathrm{~d}$-model: It only consists of the road and a sky box, in contrast to other tracks which feature details like trees, grandstands, etc. The performance of our controller "MrRacer2010" on the different tracks reflects the online learning process: The controller is best on tracks where it had the chance to complete all four phases of the online learning. It is able to outperform all other controllers on Spa and Goldstone and is even faster than a human player on Wheel2 and Forza!

In contrast, on slippery tracks like Curburgring and Brondehach or twisty circuits like Monandgo and Alpine-1 the competitors perform better. When driving alone like in our scenario, Cobostar's behaviour still generalizes very well, especially on tracks with low friction like Brondehach. But without the global knowledge of a track model, it seems unable to drive as fast as MrRacer on fast tracks like Wheel2, Forza or Goldstone which might indicate that the limited sensor range induces an upper boundary of the possible speed a controller can savely achieve when only relying on sensory information. Autopia and the controller by Jorge Muñoz employ a kind of a track model but are outperformed by MrRacer on six of the eight tracks tested. 


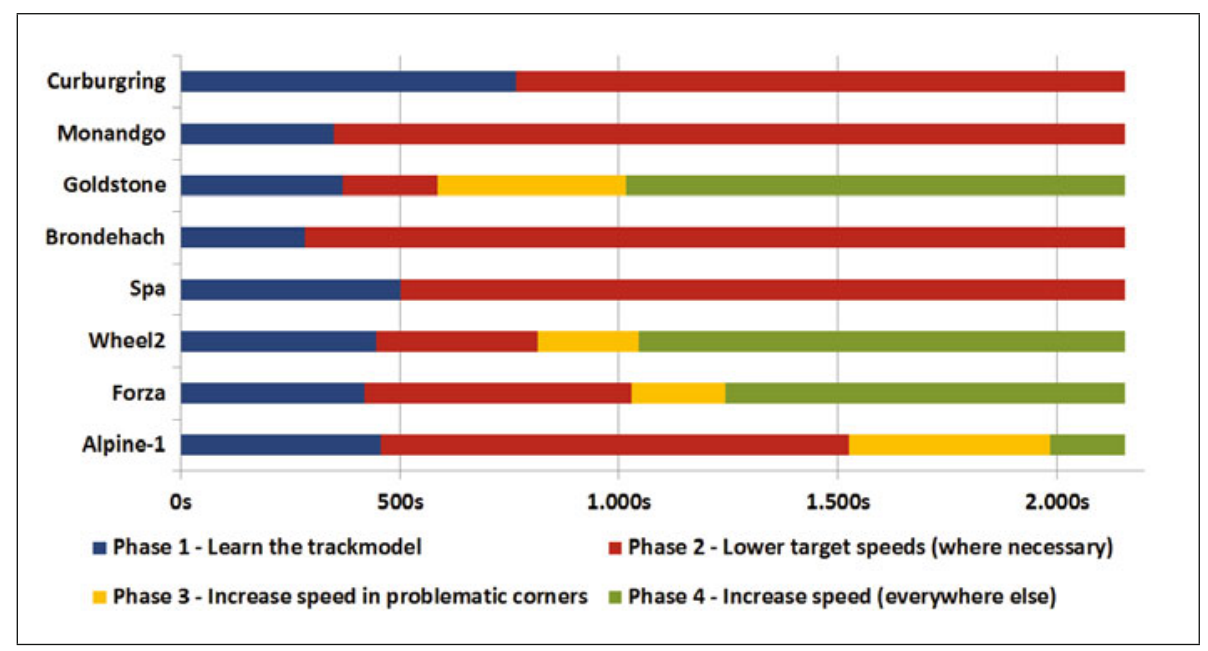

Fig. 4. Time (in seconds) allocated for the different phases of the online learning process on the various race tracks. The overall time was 100000 gameticks, which equals 2155 seconds or roughly 36 minutes.

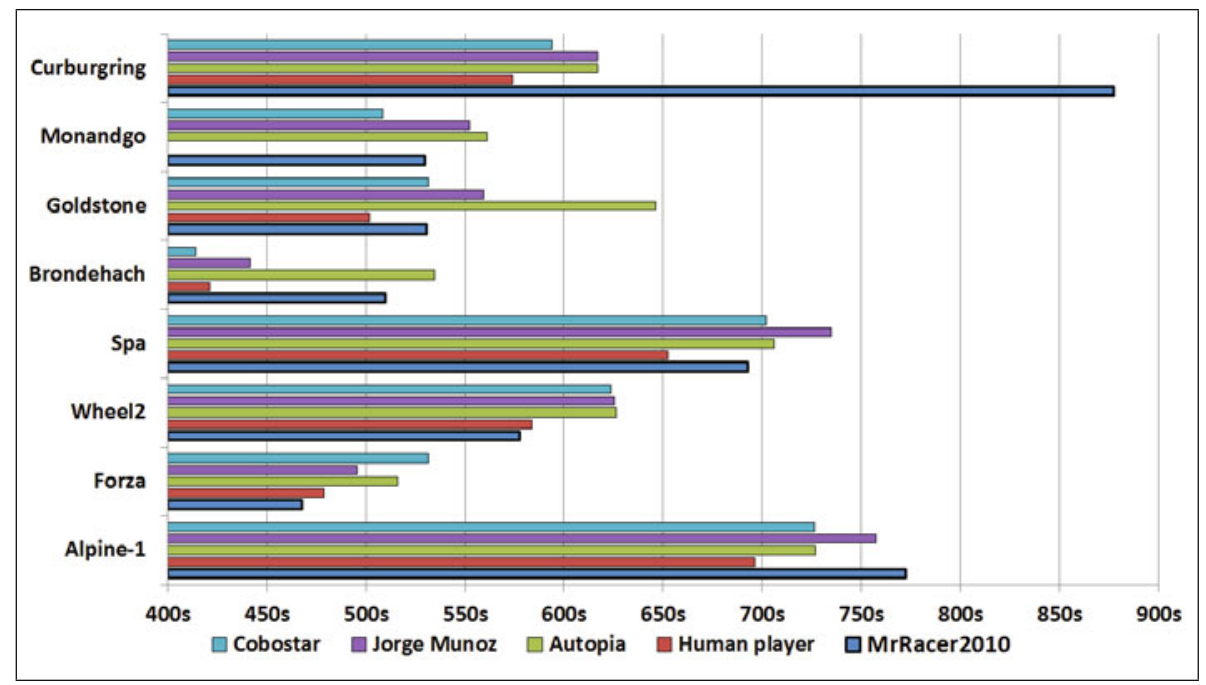

Fig. 5. Comparison of the different controllers and our human test driver. Shown are the times (in seconds) to complete 5 laps. Smaller values are better. Please note that the $\mathrm{x}$-axis starts at $400 \mathrm{~s}$.

Although our comparison shows that some problems remain to be solved, the combination of a track model, offline learning and online adaption is obviously very competitive and even able to "overtake" a human player. 


\subsection{How Does This Compare to Learning on the Track We Are Racing on?}

In the previous section we demonstrated how the behaviour learned on the track Wheel2 can be adapted online for previously unknown tracks. While the comparisons with other controllers show that the adapted behaviour is more than competitive (at least on certain tracks), an important question remains: How much faster could we drive if we learn the behaviour on the track we are racing on instead of adapting the behaviour learned on Wheel2? To answer this question, the behaviour was learned again on the track Forza, with the same optimization algorithm (CMA-ES) and setup as described in section 2 The best solution found in seven runs of the EA has a fitness of 90.8, which means it completed its fastest lap in one minute and 30.8 seconds. To drive five laps, it needs 463.31 seconds. This is only marginally faster than the solution evolved on Wheel 2 which needs one minute and 31.92 seconds for the fastest lap and 467.88 seconds to complete five laps.

At least in this case the adapted solution has nearly identical performance compared to the solution evolved on a specific track Forza. However, we have to take into account that the two tracks are of similar type, so that the advantage of pure online learning may be larger for tracks with very different characteristics.

\section{Summary and Conclusions}

By employing the undiscretized, continuous curvature of [8] directly for deciding about target speeds for any specific corner (as in opposite to first cluster them and determine a common target speed), we have established a controller that largely relies on offline optimization and is able to drive faster than all currently published controllers and even a human test driver on several tracks. This demonstrates that global knowledge of the track in form of the track model and the possibility to plan ahead enable closing the gap between bots and human drivers. We assume that this may hardly be achieved by only using local sensory information.

Our additional online adaption is still at an early stage. Observations of the online learning process show that simply setting higher target speeds to go faster is not enough: While the car might be able to complete two laps without leaving the track, it is sometimes getting closer to losing conntrol and actually driving slower than before! The simple solution would be to observe the actual lap times and only store newly adapted target speeds which lead to lower lap times. As there is only very limited time for online adaption in the car racing competition context, heuristics are needed to determine the corners which offer room for improvement. One addition that looks promising to us is the observation of the lateral speed of the car. If the lateral speed speed is high in a certain corner the car starts sliding. This can be interpreted as being close to the limit. It is questionable if one can drive any faster in such a corner and it's probably a waste of time trying to do so. On the other hand, if the lateral speed is low, things are well under control and there might be room for improvement. These are corners where a more aggressive target speed might be benefical. We have to leave it to the next championship to test these enhancements. 


\section{Acknowledgment}

The authors would like thank their tamed racing driver Thomas Fox for doing a good job as human test player, who despite of hours of training not always achieved better lap times than our controller.

\section{References}

1. Auger, A., Hansen, N.: A restart CMA evolution strategy with increasing population size. In: McKay, B., et al. (eds.) The 2005 IEEE International Congress on Evolutionary Computation (CEC 2005), vol. 2, pp. 1769-1776 (2005)

2. Butz, M., Lönneker, T.: Optimized sensory-motor couplings plus strategy extensions for the torcs car racing challenge. In: Proceedings of the IEEE Symposium on Computational Intelligence and Games, 2009, pp. 317-324 (2009)

3. Cardamone, L., Loiacono, D., Lanzi, P.L., Bardelli, A.P.: Searching for the optimal racing line using genetic algorithms. In: Proceedings of the IEEE Conference on Computational Intelligence and Games, 2010, pp. 388-394 (2010)

4. Loiacono, D., Cardamone, L., Lanzi, P.L.: Simulated car racing championship 2010: Competition software manual. Tech. Rep. 2010.8, Dipartimento di Elettronica e Informazione, Politecnico di Milano, Italy (2010)

5. Loiacono, D., Lanzi, P.L., Togelius, J., Onieva, E., Pelta, D.A., Butz, M.V., Lönneker, T.D., Cardamone, L., Perez, D., Saez, Y., Preuss, M., Quadflieg, J.: The 2009 simulated car racing championship. IEEE Transactions on Computational Intelligence and Games 2(2), 131-147 (2010)

6. Muñoz, J., Gutierrez, G., Sanchis, A.: A human-like torcs controller for the simulated car racing championship. In: Proceedings of the IEEE Conference on Computational Intelligence and Games, 2010, pp. 473-480 (2010)

7. Onieva, E., Pelta, D.A., Alonso, J., Milanés, V., Pérez, J.: A modular parametric architecture for the torcs racing engine. In: Proceedings of the IEEE Symposium on Computational Intelligence and Games, 2009, pp. 256-262 (2009)

8. Quadflieg, J., Preuss, M., Kramer, O., Rudolph, G.: Learning the track and planning ahead in a car racing controller. In: 2010 IEEE Symposium on Computational Intelligence and Games (CIG), pp. $395-402$ (2010)

9. Richards, F.J.: A Flexible Growth Function for Empirical Use. J. Exp. Bot. 10(2), 290-301 (1959)

10. Wymann, B.: The open racing car simulator (December 9, 2009), http://torcs . sourceforge.net, http://torcs.sourceforge.net 